Homology, Homotopy and Applications, vol.22(2), 2020, pp.251-258

\title{
A SIMPLE PROOF OF CURTIS' CONNECTIVITY THEOREM FOR LIE POWERS
}

\author{
SERGEI O. IVANOV, VLADISLAV ROMANOVSKII AND \\ ANDREI SEMENOV
}

(communicated by Donald M. Davis)

\begin{abstract}
We give a simple proof of Curtis' theorem: if $A_{\bullet}$ is a $k$-connected free simplicial abelian group, then $L^{n}\left(A_{\bullet}\right)$ is a $k+\left\lceil\log _{2} n\right\rceil$-connected simplicial abelian group, where $L^{n}$ is the $n$-th Lie power functor. In the proof we do not use Curtis' decomposition of Lie powers. Instead we use the ChevalleyEilenberg complex for the free Lie algebra.
\end{abstract}

\section{Introduction}

In [5] Curtis constructed a spectral sequence that converges to the homotopy groups $\pi_{*}(X)$ of a simply connected space $X$. It was described in the language of simplicial groups. This spectral sequence was an early version of the unstable Adams spectral sequence (see $[\mathbf{6}, \S 9],[\mathbf{2}]$ ). Recall that a simplicial group $G_{\bullet}$ is called $n$ connected if $\pi_{i}\left(G_{\bullet}\right)=0$ for $i \leqslant n$. For a group $G$ we denote by $\gamma_{n}(G)$ the $n$-th term of its lower central series. In order to prove the convergence of this spectral sequence, Curtis proved a theorem, which we call Curtis' connectivity theorem for lower central series. It can be formulated as follows.

Theorem ([5]). If $G_{\bullet}$ is a $k$-connected free simplicial group for $k \geqslant 0$, then the simplicial group $\gamma_{n}\left(G_{\bullet}\right)$ is $k+\left\lceil\log _{2} n\right\rceil$-connected.

Curtis gave a tricky proof of this theorem using some delicate calculations with generators in free groups. Later Rector $[\mathbf{1 1}]$ described a mod- $p$ analogue of this spectral sequence where the lower central series is replaced by the mod- $p$ lower central series. Then Quillen [10] found a more conceptual way to prove the connectivity theorem for the mod- $p$ lower central series using simplicial profinite groups. This result was enough to prove the convergence of the mod- $p$ version of the spectral sequence. Quillen reduced this connectivity theorem to an earlier result of Curtis, which we call Curtis' connectivity theorem for Lie powers. Denote by $L^{n}: \mathrm{Ab} \rightarrow \mathrm{Ab}$ the $n$-th Lie power functor. Then the theorem can be formulated as follows.

The work is supported by a grant of the Government of the Russian Federation for the state support of scientific research, agreement 14.W03.31.0030 dated 15.02.2018. The third author was also supported by "Native Towns", a social investment program of PJSC "Gazprom Neft".

Received December 17, 2019; published on May 6, 2020.

2010 Mathematics Subject Classification: 55Pxx, 55U10, 18 G30.

Key words and phrases: homotopy theory, unstable Adams spectral sequence, simplicial group, connectivity, Chevalley-Eilenberg complex.

Article available at http://dx.doi.org/10.4310/HHA.2020.v22.n2.a15

Copyright (c) 2020, International Press. Permission to copy for private use granted. 
Theorem ([4]). If $A_{\bullet}$ is a $k$-connected free simplicial abelian group, then the simplicial abelian group $L^{n}\left(A_{\bullet}\right)$ is $k+\left\lceil\log _{2} n\right\rceil$-connected.

Curtis' proof of this theorem is quite complicated and takes up most of the paper (see $[4, \S 4-7]$ ). He used the so-called "decomposition of Lie powers" into smaller functors. The decomposition is a kind of filtration on the functor $L^{n}$ (see $\left.[\mathbf{4}, \S 4]\right)$. The goal of this paper is to give a simpler proof of this theorem without the decomposition. Instead of this we use the Chevalley-Eilenberg complex for the free Lie algebra. We also generalize the statement to the case of modules over arbitrary commutative rings.

Let $R$ be a commutative ring. We say that a functor $\mathcal{F}: \operatorname{Mod}(R) \rightarrow \operatorname{Mod}(R)$ is $n$-connected if, for any $k \geqslant 0$ and any $k$-connected free simplicial module $A_{\bullet}$, the simplicial module $\mathcal{F}\left(A_{\bullet}\right)$ is $k+n$-connected. In these terms we prove the following.

Theorem. The Lie power functor $L^{n}: \operatorname{Mod}(R) \rightarrow \operatorname{Mod}(R)$ is $\left\lceil\log _{2} n\right\rceil$-connected.

We also note that this estimate of the connectivity of $L^{n}$ is best possible for $n=2^{m}$. This is an easy corollary of the description of homotopy groups of 2-restricted Lie powers in the language of lambda-algebra given in $[\mathbf{6}]$ and $[\mathbf{2}]$ (see also $[\mathbf{9}, \mathbf{8}]$ ).

Proposition. If $R=\mathbb{Z}$ or $R=\mathbb{Z} / 2$ the functor $L^{2^{n}}: \operatorname{Mod}(R) \rightarrow \operatorname{Mod}(R)$ is not $n+$ 1-connected.

Note that our proof of the main theorem is quite elementary. However, the proposition is a corollary of some non-elementary results about the lambda-algebra.

Assume that $\mathfrak{g}$ is a Lie algebra which is free as a module over the commutative ground ring $R$. By the Chevalley-Eilenberg complex of $\mathfrak{g}$ we mean the chain complex whose components are the exterior powers $\Lambda^{i} \mathfrak{g}$ and whose homology is homology of the Lie algebra with trivial coefficients $H_{*}(\mathfrak{g})$. We consider the free Lie algebra as a functor from the category of free modules to the category of Lie algebras. The free Lie algebra has a natural grading whose components are the Lie powers $L^{*}(A)=$ $\bigoplus_{n \geqslant 1} L^{n}(A)$. Here we treat Lie powers as functors from the category of free modules $L^{n}: \operatorname{FMod}(R) \rightarrow \operatorname{Mod}(R)$. The grading on the free Lie algebra induces a grading on the Chevalley-Eilenberg complex whose components give exact sequences of functors on the category of free modules:

$$
\begin{array}{r}
0 \rightarrow \Lambda^{2} \rightarrow L^{2} \rightarrow 0, \\
0 \rightarrow \Lambda^{n} \rightarrow \cdots \rightarrow \Lambda^{3} \rightarrow \operatorname{Id} \otimes L^{2} \rightarrow L^{3} \rightarrow 0, \\
0 \rightarrow \Lambda^{4} \rightarrow \Lambda^{2} \otimes L^{2} \rightarrow\left(\operatorname{Id} \otimes L^{3}\right) \oplus \Lambda^{2} L^{2} \rightarrow L^{4} \rightarrow 0, \\
\bigoplus_{\substack{k_{1}+\cdots+k_{n}=i \\
k_{1} \cdot 1+k_{2} \cdot 2+\cdots+k_{n} \cdot n=n}} \Lambda^{k_{1}} L^{1} \otimes \Lambda^{k_{2}} L^{2} \otimes \cdots \otimes \Lambda^{k_{n}} L^{n} \rightarrow \cdots \rightarrow L^{n} \rightarrow 0
\end{array}
$$

(see Corollary 2.2), where $\Lambda^{k_{s}} L^{s}$ denotes the composition of the Lie power functor and the exterior power functor. We use these complexes for induction in the proof of the main result. 


\section{Graded Chevalley-Eilenberg complex}

Throughout the paper $R$ denotes a commutative ring. All algebras, modules, simplicial modules, tensor products and exterior powers are assumed to be over $R$.

Let $\mathfrak{g}$ be a Lie algebra which is free as a module. If we tensor the ChevalleyEilenberg resolution $V_{\bullet}(\mathfrak{g})$ (see $[\mathbf{3}$, XIII $\S 7-8]$ ) on the trivial module $R$, we obtain a complex $C_{\bullet}(\mathfrak{g}) \cong R \otimes_{U \mathfrak{g}} V_{\bullet}(\mathfrak{g})$ that we call the Chevalley-Eilenberg complex. Its components are exterior powers of the Lie algebra $C_{i}(\mathfrak{g})=\Lambda^{i} \mathfrak{g}$ and the differential is given by the formula

$$
d\left(x_{1} \wedge \cdots \wedge x_{i}\right)=\sum_{s<t}(-1)^{s+t}\left[x_{s}, x_{t}\right] \wedge x_{1} \wedge \cdots \wedge \hat{x}_{s} \wedge \cdots \wedge \hat{x}_{t} \wedge \cdots \wedge x_{i} .
$$

The homology of this complex is isomorphic to the homology of the Lie algebra $\mathfrak{g}$ with trivial coefficients

$$
H_{i}(\mathfrak{g}, R)=H_{i}(C \bullet(\mathfrak{g})) .
$$

Let $\mathfrak{g}$ be a graded Lie algebra $\mathfrak{g}=\bigoplus_{n \geqslant 1} \mathfrak{g}_{n}$. By a graded Lie algebra we mean a traditional Lie algebra (not a Lie superalgebra) $\mathfrak{g}$ together with a decomposition into a direct sum of modules $\mathfrak{g}=\bigoplus_{n \geqslant 1} \mathfrak{g}_{n}$ such that $\left[\mathfrak{g}_{n}, \mathfrak{g}_{m}\right] \subseteq \mathfrak{g}_{n+m}$ for all $n, m \geqslant 1$. The degree of a homogeneous element $x \in \mathfrak{g}_{n}$ is denoted by $|x|=n$.

For $n \geqslant 1$ we consider a submodule $C_{i}^{(n)}(\mathfrak{g})$ of $C_{i}(\mathfrak{g})$ spanned by elements $x_{1} \wedge$ $\cdots \wedge x_{i}$, where $x_{1}, \ldots, x_{i}$ are homogeneous and $\left|x_{1}\right|+\cdots+\left|x_{i}\right|=n$.

$$
C_{i}^{(n)}(\mathfrak{g})=\operatorname{span}\left\{x_{1} \wedge \cdots \wedge x_{i} \in \Lambda^{i} \mathfrak{g}|| x_{1}|+\cdots+| x_{i} \mid=n\right\} .
$$

It is easy to see that $d\left(C_{i}^{(n)}(\mathfrak{g})\right) \subseteq C_{i-1}^{(n)}(\mathfrak{g})$, and hence we obtain a subcomplex $C_{\bullet}^{(n)}(\mathfrak{g})$ of $C \bullet(\mathfrak{g})$.

Proposition 2.1. Let $\mathfrak{g}=\bigoplus_{n \geqslant 1} \mathfrak{g}_{n}$ be a graded Lie algebra, where $\mathfrak{g}_{n}$ is free as a module for each $n$. Then the Chevalley-Eilenberg complex $C \bullet(\mathfrak{g})$ has a natural grading

$$
C_{\bullet}(\mathfrak{g})=\bigoplus_{n \geqslant 1} C_{\bullet}^{(n)}(\mathfrak{g})
$$

and there is a natural isomorphism

$$
C_{i}^{(n)}(\mathfrak{g}) \cong \bigoplus_{\substack{k_{1}+\cdots+k_{n}=i \\ k_{1} \cdot 1+k_{2} \cdot 2+\cdots+k_{n} \cdot n=n}} \Lambda^{k_{1}} \mathfrak{g}_{1} \otimes \Lambda^{k_{2}} \mathfrak{g}_{2} \otimes \cdots \otimes \Lambda^{k_{n}} \mathfrak{g}_{n} .
$$

Here the sum runs over the set of ordered $n$-tuples of non-negative integers $\left(k_{1}, \ldots, k_{n}\right)$ such that $k_{1}+\cdots+k_{n}=i$ and $k_{1} \cdot 1+k_{2} \cdot 2+\cdots+k_{n} \cdot n=n$.

Proof. For any modules $A, B$ there is an isomorphism $\Lambda^{i}(A \oplus B) \cong \bigoplus_{k+l=i} \Lambda^{k}(A) \otimes$ $\Lambda^{l}(B)$. By induction we obtain the isomorphism

$$
\Lambda^{i}\left(\bigoplus_{s=1}^{N} A_{s}\right) \cong \bigoplus_{k_{1}+\cdots+k_{N}=i} \Lambda^{k_{1}} A_{1} \otimes \cdots \otimes \Lambda^{k_{N}} A_{N} .
$$

Using the fact that the exterior power commutes with direct limits, we obtain the 
isomorphism, for any infinite sequence of modules $A_{1}, A_{2}, \ldots$,

$$
\Lambda^{i}\left(\bigoplus_{s=1}^{\infty} A_{i}\right) \cong \bigoplus_{k_{1}+k_{2}+\cdots=i} \Lambda^{k_{1}} A_{1} \otimes \Lambda^{k_{2}} A_{2} \otimes \cdots
$$

Here we consider only those sequences of non-negative integers $k_{1}, k_{2}, \ldots$ with only finitely many non-zero elements, and hence, each summand in the sum is a finite tensor product.

Take $A_{n}=\mathfrak{g}_{n}$. If we have an element $x_{1} \wedge \cdots \wedge x_{i}$ with homogeneous $x_{s} \in \mathfrak{g}$ from the $R$-submodule corresponding to a summand $\Lambda^{k_{1}} \mathfrak{g}_{1} \otimes \Lambda^{k_{2}} \mathfrak{g}_{2} \otimes \cdots$, then $\left|x_{1}\right|+\cdots+$ $\left|x_{n}\right|=k_{1} \cdot 1+k_{2} \cdot 2+\cdots$. The assertion follows.

Let $A$ be a free module. We denote by $L^{*}(A)$ the free Lie algebra generated by $A$ For any basis $\left(a_{s}\right)$ of $A, L^{*}(A)$ is isomorphic to the free Lie algebra generated by the family $\left(a_{s}\right)$. The Lie algebra $L^{*}(A)$ is free as a module (see [13], [12, Cor. 0.10]). Its enveloping algebra is the tensor algebra $T^{*}(A)$. The map $L^{*}(A) \rightarrow T^{*}(A)$ is injective $\left[12\right.$, Cor. 0.3]. Hence, $L^{*}(A)$ can be described in terms of the tensor algebra. Consider the tensor algebra $T^{*}(A)$ as a Lie algebra with respect to the commutator. Then $L^{*}(A)$ can be described as the Lie subalgebra of $T^{*}(A)$ generated by $A$ (see also $[6, \S 7.4]$ ).

The Lie algebra $L^{*}(A)$ has a natural grading

$$
L^{*}(A)=\bigoplus_{n=1}^{\infty} L^{n}(A)
$$

where $L^{n}(A)$ is generated by $n$-fold commutators. Equivalently $L^{n}(A)$ can be described using the embedding into the tensor algebra as $L^{n}(A)=L(A) \cap T^{n}(A)$. The homology of the free Lie algebra $L^{*}(A)$ can be described as follows $H_{i}\left(L^{*}(A)\right)=0$ for $i>1$ and $H_{1}\left(L^{*}(A)\right)=A$. For simplicity we set

$$
\mathrm{C}_{\bullet}^{(n)}(A):=C_{\bullet}^{(n)}\left(L^{*}(A)\right) .
$$

All these constructions are natural in $A$. Denote by $L^{n}$ the $n$-th Lie power functor from the category of free modules to the category of modules

$$
L^{n}: \operatorname{FMod}(R) \longrightarrow \operatorname{Mod}(R) .
$$

Moreover, we treat $\mathrm{C}_{\bullet}^{(n)}$ as a complex in the category of functors $\mathrm{FMod}(R) \rightarrow \operatorname{Mod}(R)$. Then Proposition 2.1 implies the following corollary.

Corollary 2.2. For $n \geqslant 2$ the complex $\mathrm{C}_{\bullet}^{(n)}$ of functors $\mathrm{FMod}(R) \rightarrow \operatorname{Mod}(R)$ is acyclic and has the following components

$$
\mathrm{C}_{i}^{(n)}=\bigoplus_{\begin{array}{c}
k_{1}+\cdots+k_{n}=i \\
k_{1} \cdot 1+k_{2} \cdot 2+\cdots+k_{n} \cdot n=n
\end{array}} \Lambda^{k_{1}} L^{1} \otimes \Lambda^{k_{2}} L^{2} \otimes \cdots \otimes \Lambda^{k_{n}} L^{n},
$$

where $\Lambda^{k_{s}} L^{s}$ denotes the composition of the Lie power functor and the exterior power functor. Here the sum runs over the set of ordered n-tuples of non-negative integers $\left(k_{1}, \ldots, k_{n}\right)$ such that $k_{1}+\cdots+k_{n}=i$ and $k_{1} \cdot 1+k_{2} \cdot 2+\cdots+k_{n} \cdot n=n$. 
Remark 2.3. Note that $\mathrm{C}_{i}^{(n)}=0$ for $i \notin\{1, \ldots, n\}$, and that there are isomorphisms $\mathrm{C}_{n}^{(n)}=\Lambda^{n}$ and $\mathrm{C}_{1}^{(n)}=L^{n}$. In other words $\mathrm{C}_{\bullet}^{(n)}$ is an exact sequence that connects $\Lambda^{n}$ and $L^{n}$

$$
\mathrm{C}_{\bullet}^{(n)}: \quad 0 \rightarrow \Lambda^{n} \rightarrow \cdots \rightarrow L^{n} \rightarrow 0
$$

\section{Connectivity of functors}

For $n \geqslant 0$ we say that a simplicial module $A_{\bullet}$ is $n$-connected, if $\pi_{i}\left(A_{\bullet}\right)=0$ for $i \leqslant n$.

Lemma 3.1. Let $A_{\bullet}$ be an $n$-connected simplicial module and $B_{\bullet}$ an $m$-connected free simplicial module. Then $A_{\bullet} \otimes B \bullet$ is $n+m+1$-connected.

Proof. Consider their component-wise tensor product $A_{\bullet} \otimes B_{\bullet}$. The Eilenberg-Zilber theorem implies that $\pi_{i}\left(A_{\bullet} \otimes B_{\bullet}\right) \cong H_{i}\left(N A_{\bullet} \otimes N B_{\bullet}\right)$, where $N C_{\bullet}$ denotes the Moore complex of $C_{\bullet}$. Since $N_{i} B_{\bullet}$ is a direct summand of $B_{i}$, it is a projective module. This gives the following variant of the Künneth spectral sequence:

$$
E_{p q}^{2}=\bigoplus_{s+t=q} \operatorname{Tor}_{p}^{R}\left(\pi_{s}\left(A_{\bullet}\right), \pi_{t}\left(B_{\bullet}\right)\right) \Rightarrow \pi_{p+q}\left(A_{\bullet} \otimes B_{\bullet}\right) .
$$

If $s+t \leqslant n+m+1$, then either $s<n+1$ or $t<m+1$. Hence $E_{p q}^{2}=0$ for $p+q \leqslant$ $n+m+1$. Therefore, $A_{\bullet} \otimes B_{\bullet}$ is $n+m+1$-connected.

A functor from the category of modules to itself

$$
\mathcal{F}: \operatorname{Mod}(R) \longrightarrow \operatorname{Mod}(R)
$$

is said to be $n$-connected if, for any $k \geqslant 0$ and any $k$-connected free simplicial module $A_{\bullet}$, the simplicial module $\mathcal{F}\left(A_{\bullet}\right)$ is $k+n$-connected.

Lemma 3.2. Let $\mathcal{F}: \operatorname{Mod}(R) \rightarrow \operatorname{Mod}(R)$ be an $n$-connected functor and $\mathcal{G}: \operatorname{Mod}(R) \rightarrow$ $\operatorname{Mod}(R)$ an $m$-connected functor. Assume that $\mathcal{G}$ sends free modules to free modules. Then the composition $\mathcal{F G}$ is $n+m$-connected and the tensor product $\mathcal{F} \otimes \mathcal{G}$ is $n+m+1$-connected.

Proof. The fact about the composition is obvious. The fact about the tensor product follows from Lemma 3.1.

Lemma 3.3. Let

$$
0 \rightarrow \mathcal{F}_{n} \rightarrow \cdots \rightarrow \mathcal{F}_{1} \rightarrow \mathcal{F}_{0} \rightarrow \mathcal{G} \rightarrow 0
$$

be an exact sequence of functors such that $\mathcal{F}_{i}$ is $n-i$-connected. Then $\mathcal{G}$ is $n$ connected.

Proof. The proof is by induction. For $n=0$ this is obvious. Assume that $n \geqslant 1$ and that the statement holds for smaller numbers. Set $\mathcal{H}:=\operatorname{Ker}\left(\mathcal{F}_{0} \rightarrow \mathcal{G}\right)$. Then by the induction hypothesis $\mathcal{H}$ is $n-1$-connected. The long exact sequence

$$
\cdots \rightarrow \pi_{i}\left(\mathcal{H}\left(A_{\bullet}\right)\right) \rightarrow \pi_{i}\left(\mathcal{F}_{0}\left(A_{\bullet}\right)\right) \rightarrow \pi_{i}\left(\mathcal{G}\left(A_{\bullet}\right)\right) \rightarrow \pi_{i-1}\left(\mathcal{H}\left(A_{\bullet}\right)\right) \rightarrow \cdots
$$

implies that $\mathcal{G}$ is $n$-connected. 
Proposition 3.4. The exterior power functor $\Lambda^{n}$ is $n$-1-connected.

Proof. The décalage formula [7, Prop. 4.3.2.1] for exterior and divided powers $\Lambda^{n}, \Gamma^{n}$ gives a homotopy equivalence, for any free simplicial module $B_{\bullet}$,

$$
\Lambda^{n}\left(B_{\bullet}[1]\right) \sim \Gamma^{n}\left(B_{\bullet}\right)[n] .
$$

Any 0 -connected free simplicial module $A_{\bullet}$ is homotopy equivalent to a simplicial module of the form $B_{\bullet}[1]$, where $B_{\bullet}$ is also a free simplicial module (it follows from the same fact for non-negatively graded chain complexes). Moreover, if $A_{\bullet}$ is $k$-connected, we can chose $B_{\bullet}$ so that $B_{i}=0$ for $i \leqslant k-1$. Hence $\pi_{i}\left(\Lambda^{n}\left(A_{\bullet}\right)\right)=\pi_{i}\left(\Lambda^{n}\left(B_{\bullet}[1]\right)\right)=$ $\pi_{i-n}\left(\Gamma^{n}\left(B_{\bullet}\right)\right)=0$ for $i \leqslant k+n-1$.

Lemma 3.5. For any two sequences of positive integer numbers $u_{1}, \ldots, u_{m}$ and $v_{1}, \ldots, v_{m}$ the following inequality holds

$$
\sum_{s=1}^{m}\left(u_{s}+\log _{2} v_{s}\right) \geqslant 1+\log _{2}\left(\sum_{s=1}^{m} u_{s} v_{s}\right) .
$$

Proof. It is easy to prove by induction that $\prod_{s=1}^{m} 2^{u_{s}} v_{s} \geqslant 2 \sum_{s=1}^{m} u_{s} v_{s}$. If we apply logarithms, we obtain the required statement.

Theorem 3.6. The Lie power functor $L^{n}$ is $\left\lceil\log _{2} n\right\rceil$-connected.

Proof. The proof is by induction. For $n=1$ we have $L^{1}=\mathrm{Id}$ and this is obvious. Assume that $n \geqslant 2$ and that the statement holds for all smaller numbers. Consider the acyclic chain complex $\mathrm{C}_{\bullet}^{(n)}$ (Corollary 2.2). Using Lemma 3.3 we obtain that it is enough to check that the functor

$$
\mathrm{C}_{i}^{(n)}=\bigoplus_{\begin{array}{c}
k_{1}+\cdots+k_{n}=i \\
k_{1} \cdot 1+k_{2} \cdot 2+\cdots+k_{n} \cdot n=n
\end{array}} \Lambda^{k_{1}} L^{1} \otimes \Lambda^{k_{2}} L^{2} \otimes \cdots \otimes \Lambda^{k_{n}} L^{n}
$$

is $\left\lceil\log _{2} n\right\rceil$-connected for $i \geqslant 2$. It is enough to prove this for each summand.

Fix an $n$-tuple of $\left(k_{1}, \ldots, k_{n}\right)$ such that $k_{1}+\cdots+k_{n}=i \geqslant 2$ and $k_{1} \cdot 1+k_{2} \cdot 2+$ $\cdots+k_{n} \cdot n=n$. Note that $i \geqslant 2$ implies $k_{n}=0$. Some of the numbers $k_{j}$ are equal to zero. Denote by $j_{1}, \ldots, j_{m}$ the indexes corresponding to non-zero numbers $k_{j_{s}} \neq 0$. By Lemma 3.2 the functor $\Lambda^{k_{j}} L^{j}$ is $k_{j}-1+\left\lceil\log _{2} j\right\rceil$-connected for $j<n$. Then again by Lemma 3.2 the tensor product

$$
\Lambda^{k_{1}} L^{1} \otimes \Lambda^{k_{2}} L^{2} \otimes \cdots \otimes \Lambda^{k_{n}} L^{n}
$$

is $\sum_{s=1}^{m}\left(k_{j_{s}}-1+\left\lceil\log _{2} j_{s}\right\rceil\right)+m-1$-connected. Using Lemma 3.5 we obtain

$$
\sum_{s=1}^{m}\left(k_{j_{s}}-1+\log _{2} j_{s}\right)+m-1=\sum_{s=1}^{m}\left(k_{j_{s}}+\log _{2} j_{s}\right)-1 \geqslant \log _{2} n .
$$

The assertion follows.

\section{Connectivity of $L^{2^{n}}$}

For $k \geqslant 0$ we denote by $R[k+1]$ the chain complex concentrated in degree $k+1$, whose $k+1$-st component is equal to $R$. The corresponding Dold-Kan simplicial module is denoted by $K(R, k+1)$. Note that $K(R, k+1)$ is a $k$-connected free simplicial module. 
Proposition 4.1. Let $R=\mathbb{Z}$ or $R=\mathbb{Z} / 2$ and $n \geqslant 0$. Then $L^{2^{n}}$ is not $n+1$-connected. Moreover, for any $k \geqslant 0$

$$
\pi_{n+1+k}\left(L^{2^{n}}(K(R, k+1)) \neq 0 .\right.
$$

Proof. (1) Let $R=\mathbb{Z} / 2$. Fix $k$ and set $V_{\bullet}=K(\mathbb{Z} / 2, k+1)$. Denote by $L_{\text {res }}^{n}$ : $\operatorname{Vect}(\mathbb{Z} / 2) \rightarrow$ $\operatorname{Vect}(\mathbb{Z} / 2)$ the 2 -restricted Lie power functor (see $[\mathbf{1}, \S 2.7],[6, \S 7.5]$ ). The homotopy groups $\pi_{*}\left(L_{\text {res }}^{2^{n}}\left(V_{\bullet}\right)\right)$ are described in terms of the lambda-algebra in [6, Th. 8.8] (see also [2] and the discussion after Theorem 7.11 in [6]):

$$
\pi_{i+k+1}\left(L_{\text {res }}^{2^{n}}\left(V_{\bullet}\right)\right) \cong \Lambda^{i, n}(k+2),
$$

where $\Lambda^{i, n}(k+2)$ denotes the vector sub-space of the lambda algebra $\Lambda$ with the basis given by compositions $\lambda_{i_{1}} \cdots \lambda_{i_{n}}$, where $i_{s+1} \leqslant 2 i_{s}, i_{1}+\cdots+i_{n}=i$ and $i_{1} \leqslant k+2$. In particular, $\lambda_{1}^{n} \in \Lambda^{n, n}(k+2) \neq 0$. Hence

$$
\pi_{n+1+k}\left(L_{\text {res }}^{2^{n}}\left(V_{\bullet}\right)\right) \neq 0 .
$$

For an arbitrary simplicial Lie algebra $\mathfrak{g}_{\bullet}$ and $t \geqslant 1$ we define the map $\tilde{\lambda}_{1}: \mathfrak{g}_{t} \rightarrow$ $\mathfrak{g}_{t+1}$ by the formula $\tilde{\lambda}_{1}(x)=\left[s_{0} x, s_{1} x\right]$, where $s_{0}, s_{1}$ are the degeneracy maps. Denote by $\mathfrak{i}_{k+1}$ the unit of $\left(V_{\bullet}\right)_{k+1}=R$. Then $\tilde{\lambda}_{1}^{n}\left(\mathfrak{i}_{k+1}\right) \in\left(L\left(V_{\bullet}\right)\right)_{n+k+1}$ is the element representing $\lambda_{1}^{n} \in \Lambda^{n, n}(k+2) \cong \pi_{n+1+k}\left(L_{\text {res }}^{2^{n}}\left(V_{\bullet}\right)\right)$ (see [6, Prop. 8.6]). By the definition of $\tilde{\lambda}_{1}$, the element $\tilde{\lambda}_{1}^{n}\left(\mathfrak{i}_{k+1}\right)$ lies in the unrestricted part $L^{2^{n}}\left(V_{\bullet}\right)$ of $L_{\text {res }}^{2^{n}}\left(V_{\bullet}\right)$. Therefore $\tilde{\lambda}_{1}^{n}\left(\mathfrak{i}_{k+1}\right)$ represents a nontrivial element of $\pi_{n+1+k}\left(L^{2^{n}}\left(V_{\bullet}\right)\right)$ and hence

$$
\pi_{n+1+k}\left(L^{2^{n}}\left(V_{\bullet}\right)\right) \neq 0 .
$$

(2) Now assume that $R=\mathbb{Z}$ and set $A_{\bullet}=K(\mathbb{Z}, k+1)$. We denote by $L_{\mathbb{Z} / 2}^{*}$ the Lie power functor over $\mathbb{Z} / 2$, which we already discussed, and by $L_{\mathbb{Z}}^{*}$ the Lie power functor over $\mathbb{Z}$. Then for any free abelian group $A$ we have $L_{\mathbb{Z}}^{*}(A) \otimes \mathbb{Z} / 2 \cong L_{\mathbb{Z} / 2}^{*}(A \otimes \mathbb{Z} / 2)$. The universal coefficient theorem gives the following short exact sequence

$$
0 \longrightarrow \pi_{i}\left(L_{\mathbb{Z}}^{2^{n}}\left(A_{\bullet}\right)\right) \otimes \mathbb{Z} / 2 \longrightarrow \pi_{i}\left(L_{\mathbb{Z} / 2}^{2^{n}}\left(V_{\bullet}\right)\right) \longrightarrow \operatorname{Tor}_{1}^{\mathbb{Z}}\left(\pi_{i-1}\left(L_{\mathbb{Z}}^{2^{n}}\left(A_{\bullet}\right)\right), \mathbb{Z} / 2\right) \longrightarrow 0 .
$$

Since the functor $L_{\mathbb{Z}}^{2^{n}}$ is $n$-connected, $\pi_{n+k}\left(L_{\mathbb{Z}}^{2^{n}}\left(A_{\bullet}\right)\right)=0$. Therefore

$$
\pi_{n+1+k}\left(L_{\mathbb{Z}}^{2^{n}}\left(A_{\bullet}\right)\right) \otimes \mathbb{Z} / 2 \cong \pi_{n+1+k}\left(L_{\mathbb{Z} / 2}^{2^{n}}\left(V_{\bullet}\right)\right)
$$

We already proved that $\pi_{n+1+k}\left(L_{\mathbb{Z} / 2}^{2^{n}}\left(V_{\bullet}\right)\right) \neq 0$. Hence $\pi_{n+1+k}\left(L_{\mathbb{Z}}^{2^{n}}\left(A_{\bullet}\right)\right) \neq 0$.

Remark 4.2. Proposition 4.1 can be also deduced from results of D. Leibowitz [8] or from unpublished results of R. Mikhailov [9], where he describes all derived functors in the sense of Dold-Puppe of Lie powers in the case $R=\mathbb{Z}$.

\section{References}

[1] Yu.A. Bahturin. Identical relations in Lie algebras. VNU Science Press, b.v., Utrecht, 1987.

[2] A.K. Bousfield, E.B. Curtis, D.M. Kan, D.G. Quillen, D.L. Rector, J.W. Schlesinger. The mod- $p$ lower central series and the Adams spectral sequence, Topology 5 (1966), 331-342. 
[3] H. Cartan, S. Eilenberg. Homological algebra. Princeton University Press, Princeton, N.J., 1956.

[4] E.B. Curtis. Lower central series of semi-simplicial complexes, Topology 2 (1963), 159-171.

[5] E.B. Curtis. Some relations between homotopy and homology, Ann. of Math. 82 (1965), 386-413.

[6] E.B. Curtis. Simplicial Homotopy Theory, Adv. Math. 6 (1971), 107-209.

[7] L. Illusie. Complexe cotangent et déformations. I, Lecture Notes in Mathematics, Vol. 239, Springer-Verlag, Berlin, New York, 1971.

[8] D. Leibowitz. The $E^{1}$ term of the lower central series spectral sequence for the homotopy of spaces, Brandeis University, Ph.D. thesis (1972).

[9] R. Mikhailov. Homotopy theory of Lie functors. arXiv:1808.00681.

[10] D.G. Quillen. An application of simplicial profinite groups. Comment. Math. Helv. 44 (1969), 45-60.

[11] D.L. Rector. An unstable Adams spectral sequence, Topology 5 (1966), 343346.

[12] C. Reutenauer. Free Lie algebras, Oxford University Press, 1993.

[13] A.I. Shirshov. Subalgebras of free Lie algebras, Mat. Sb. (N.S.) 33(75), (1953), 441-452.

Sergei O. Ivanov ivanov.s.o.1986@gmail.com

Laboratory of Modern Algebra and Applications, St. Petersburg State University, 14th Line, 29b, Saint Petersburg, 199178 Russia

Vladislav Romanovskii Romanovskiy.vladislav.00@mail.ru

Laboratory of Modern Algebra and Applications, St. Petersburg State University, 14th Line, 29b, Saint Petersburg, 199178 Russia

Andrei Semenov asemenov.spb.56@gmail.com

Chebyshev Laboratory, St. Petersburg State University, 14th Line, 29b, Saint Petersburg, 199178 Russia 